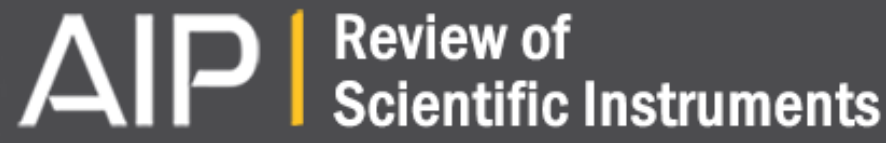

Overview of diagnostic performance and results for the first operation phase in Wendelstein 7-X (invited)

M. Krychowiak, A. Adnan, A. Alonso, T. Andreeva, J. Baldzuhn, T. Barbui, M. Beurskens, W. Biel, C. Biedermann, B. D. Blackwell, H. S. Bosch, S. Bozhenkov, R. Brakel, T. Bräuer, B. Brotas de Carvalho, R. Burhenn, B. Buttenschön, A. Cappa, G. Cseh, A. Czarnecka, A. Dinklage, P. Drews, A. Dzikowicka, F. Effenberg, M. Endler, V. Erckmann, T. Estrada, O. Ford, T. Fornal, H. Frerichs, G. Fuchert, J. Geiger, O. Grulke, J. H. Harris, H. J. Hartfuß, D. Hartmann, D. Hathiramani, M. Hirsch, U. Höfel, S. Jabłoński, M. W. Jakubowski, J. Kaczmarczyk, T. Klinger, S. Klose, J. Knauer, G. Kocsis, R. König, P. Kornejew, A. KrämerFlecken, N. Krawczyk, T. Kremeyer, I. Książek, M. Kubkowska, A. Langenberg, H. P. Laqua, M. Laux, S. Lazerson, Y. Liang, S. C. Liu, A. Lorenz, A. O. Marchuk, S. Marsen, V. Moncada, D. Naujoks, H. Neilson, O. Neubauer, U. Neuner, H. Niemann, J. W. Oosterbeek, M. Otte, N. Pablant, E. Pasch, T. Sunn Pedersen, F. Pisano, K. Rahbarnia, L. Ryć, O. Schmitz, S. Schmuck, W. Schneider, T. Schröder, H. Schuhmacher, B. Schweer, B. Standley, T. Stange, L. Stephey, J. Svensson, T. Szabolics, T. Szepesi, H. Thomsen, J.-M. Travere, H. Trimino Mora, H. Tsuchiya, G. M. Weir, U. Wenzel, A. Werner, B. Wiegel, T. Windisch, R. Wolf, G. A. Wurden, D. Zhang, A. Zimbal, S. Zoletnik, and W7-X Team

Citation: Review of Scientific Instruments 87, 11D304 (2016); doi: 10.1063/1.4964376 View online: http://dx.doi.org/10.1063/1.4964376

View Table of Contents: http://scitation.aip.org/content/aip/journal/rsi/87/11 ?ver=pdfcov Published by the AIP Publishing

\section{Articles you may be interested in}

A near infra-red video system as a protective diagnostic for electron cyclotron resonance heating operation in the Wendelstein $7-X$ stellarator

Rev. Sci. Instrum. 86, 113504 (2015); 10.1063/1.4935686

Diagnostics development for quasi-steady-state operation of the Wendelstein 7-X stellarator (invited)a)

Rev. Sci. Instrum. 83, $10 \mathrm{D} 730$ (2012); 10.1063/1.4733531

Diagnostics design for steady-state operation of the Wendelstein 7-X stellaratora)

Rev. Sci. Instrum. 81, 10E133 (2010); 10.1063/1.3483210

Diagnostic developments for quasicontinuous operation of the Wendelstein 7-X stellaratora) Rev. Sci. Instrum. 79, 10F337 (2008); 10.1063/1.2964998

Video Diagnostic for W7-X Stellarator

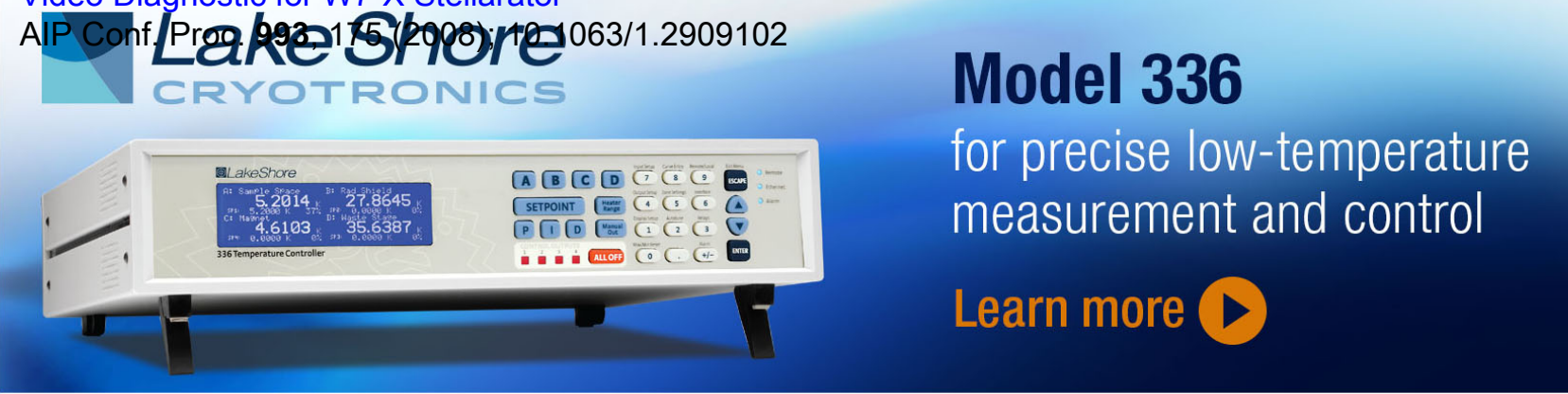




\title{
Overview of diagnostic performance and results for the first operation phase in Wendelstein 7-X (invited)
}

\author{
M. Krychowiak, ${ }^{1, a)}$ A. Adnan, ${ }^{1}$ A. Alonso, ${ }^{2}$ T. Andreeva, ${ }^{1}$ J. Baldzuhn, ${ }^{1}$ T. Barbui, ${ }^{3}$ \\ M. Beurskens, ${ }^{1}$ W. Biel, ${ }^{4}$ C. Biedermann, ${ }^{1}$ B. D. Blackwell, ${ }^{5}$ H. S. Bosch, ${ }^{1}$ S. Bozhenkov, ${ }^{1}$ \\ R. Brakel, ${ }^{1}$ T. Bräuer, ${ }^{1}$ B. Brotas de Carvalho, ${ }^{6}$ R. Burhenn, ${ }^{1}$ B. Buttenschön, ${ }^{1}$ A. Cappa, ${ }^{2}$ \\ G. Cseh, ${ }^{7}$ A. Czarnecka, ${ }^{8}$ A. Dinklage, ${ }^{1}$ P. Drews, ${ }^{4}$ A. Dzikowicka, ${ }^{9}$ F. Effenberg, ${ }^{3}$ \\ M. Endler, ${ }^{1}$ V. Erckmann, ${ }^{1}$ T. Estrada, ${ }^{2}$ O. Ford,${ }^{1}$ T. Fornal, ${ }^{8}$ H. Frerichs, ${ }^{3} \mathrm{G}$. Fuchert, ${ }^{1}$ \\ J. Geiger, ${ }^{1}$ O. Grulke, ${ }^{1}$ J. H. Harris, ${ }^{10}$ H. J. Hartfuß, ${ }^{1}$ D. Hartmann, ${ }^{1}$ D. Hathiramani, ${ }^{1}$ \\ M. Hirsch, ${ }^{1}$ U. Höfel, ${ }^{1}$ S. Jabłoński, ${ }^{8}$ M. W. Jakubowski, ${ }^{1}$ J. Kaczmarczyk, ${ }^{8}$ T. Klinger, ${ }^{1}$ \\ S. Klose, ${ }^{1}$ J. Knauer, ${ }^{1}$ G. Kocsis, ${ }^{7}$ R. König, ${ }^{1}$ P. Kornejew, ${ }^{1}$ A. Krämer-Flecken, ${ }^{4}$ \\ N. Krawczyk, ${ }^{8}$ T. Kremeyer, ${ }^{3}$ I. Książek, ${ }^{11}$ M. Kubkowska, ${ }^{8}$ A. Langenberg, ${ }^{1}$ H. P. Laqua, ${ }^{1}$ \\ M. Laux, ${ }^{1}$ S. Lazerson, ${ }^{12}$ Y. Liang, ${ }^{4}$ S. C. Liu, ${ }^{4}$ A. Lorenz, ${ }^{1}$ A. O. Marchuk, ${ }^{4}$ S. Marsen, ${ }^{1}$ \\ V. Moncada, ${ }^{13}$ D. Naujoks, ${ }^{1}$ H. Neilson, ${ }^{12}$ O. Neubauer, ${ }^{4}$ U. Neuner, ${ }^{1}$ H. Niemann, ${ }^{1} \mathrm{~J}$. \\ W. Oosterbeek, ${ }^{14}$ M. Otte, ${ }^{1}$ N. Pablant, ${ }^{12}$ E. Pasch, ${ }^{1}$ T. Sunn Pedersen, ${ }^{1}$ F. Pisano, ${ }^{15}$ \\ K. Rahbarnia, ${ }^{1}$ L. Ryć, ${ }^{8}$ O. Schmitz, ${ }^{3}$ S. Schmuck, ${ }^{16}$ W. Schneider, ${ }^{1}$ T. Schröder, ${ }^{1}$ \\ H. Schuhmacher, ${ }^{17}$ B. Schweer, ${ }^{4}$ B. Standley, ${ }^{1}$ T. Stange, ${ }^{1}$ L. Stephey, ${ }^{3}$ J. Svensson, ${ }^{1}$ \\ T. Szabolics, ${ }^{7}$ T. Szepesi, ${ }^{7}$ H. Thomsen, ${ }^{1}$ J.-M. Travere, ${ }^{13}$ H. Trimino Mora, ${ }^{1}$ H. Tsuchiya, ${ }^{18}$ \\ G. M. Weir, ${ }^{1}$ U. Wenzel, ${ }^{1}$ A. Werner, ${ }^{1}$ B. Wiegel, ${ }^{17}$ T. Windisch, ${ }^{1}$ R. Wolf, ${ }^{1}$ G. A. Wurden, ${ }^{19}$ \\ D. Zhang, ${ }^{1}$ A. Zimbal, ${ }^{17}$ S. Zoletnik, ${ }^{7}$ and W7-X Team ${ }^{1, b)}$ \\ ${ }^{1}$ Max Planck Institute for Plasma Physics, 17491 Greifswald, Germany \\ ${ }^{2}$ Laboratorio Nacional de Fusión, CIEMAT, Avenida Complutense, Madrid, Spain \\ ${ }^{3}$ University of Wisconsin, Engineering Drive, Madison, Wisconsin 53706, USA \\ ${ }^{4}$ Forschungszentrum Jülich GmbH, Institut für Energie- und Klimaforschung - Plasmaphysik, \\ Partner of the Trilateral Euregio Cluster (TEC), 52425 Jülich, Germany \\ ${ }^{5}$ Australian National University, Acton ACT, 2601 Canberra, Australia \\ ${ }^{6}$ Instituto de Plasmas e Fusao Nuclear, Avenue Rovisco Pais 1, 1049-001 Lisboa, Portugal \\ ${ }^{7}$ Wigner Research Centre for Physics, Konkoly Thege 29-33, H-1121 Budapest, Hungary \\ ${ }^{8}$ Institute of Plasma Physics and Laser Microfusion, Hery Street 23, 01-497 Warsaw, Poland \\ ${ }^{9}$ University of Szczecin, al. Papieża Jana Pawta II 22A, Szczecin, Poland \\ ${ }^{10}$ Oak Ridge National Laboratory, Oak Ridge, Tennessee 37831, USA \\ ${ }^{11}$ Opole University, pl. Kopernika 11a, 45-040 Opole, Poland \\ ${ }^{12}$ Princeton Plasma Physics Laboratory, Princeton, New Jersey 08543, USA \\ ${ }^{13}$ CEA, IRFM, F-13108 Saint-Paul-lez-Durance, France \\ ${ }^{14}$ Eindhoven University of Technology, P.O. Box 513, 5600 MB Eindhoven, The Netherlands \\ ${ }^{15}$ University of Cagliari, Via Università, 40, 09124 Cagliari, Italy \\ ${ }^{16}$ Culham Science Centre, Abingdon OX14 3DB, United Kingdom \\ ${ }^{17}$ Physikalisch-Technische Bundesanstalt, Bundesallee 100, 38116 Braunschweig, Germany \\ ${ }^{18}$ NIFS National Institute for Fusion Science, 322-6 Oroshi-cho, Toki 509-5292, Japan \\ ${ }^{19}$ Los Alamos National Laboratory, Los Alamos, New Mexico 87545, USA
}

(Presented 6 June 2016; received 23 June 2016; accepted 21 September 2016; published online 27 October 2016)

Wendelstein 7-X, a superconducting optimized stellarator built in Greifswald/Germany, started its first plasmas with the last closed flux surface (LCFS) defined by 5 uncooled graphite limiters in December 2015. At the end of the 10 weeks long experimental campaign (OP1.1) more than 20 independent diagnostic systems were in operation, allowing detailed studies of many interesting plasma phenomena. For example, fast neutral gas manometers supported by video cameras (including one fast-frame camera with frame rates of tens of $\mathrm{kHz}$ ) as well as visible cameras with different interference filters, with field of views covering all ten half-modules of the stellarator, discovered a MARFE-like radiation zone on the inboard side of machine module 4. This structure is presumably triggered by an inadvertent plasma-wall interaction in module 4 resulting in a high impurity influx that terminates some discharges by radiation cooling. The main plasma parameters achieved in OP1.1 exceeded predicted values in discharges of a length reaching $6 \mathrm{~s}$. Although OP1.1 is characterized by

\footnotetext{
Note: Invited paper, published as part of the Proceedings of the 21st Topical Conference on High-Temperature Plasma Diagnostics, Madison, Wisconsin, USA, June 2016.

a) Author to whom correspondence should be addressed. Electronic mail: cak@ipp.mpg.de

b) Members of the W7-X Team are listed in Nucl. Fusion 53, 126001 (2013).
} 
short pulses, many of the diagnostics are already designed for quasi-steady state operation of $30 \mathrm{~min}$ discharges heated at $10 \mathrm{MW}$ of ECRH. An overview of diagnostic performance for OP1.1 is given, including some highlights from the physics campaigns. [http://dx.doi.org/10.1063/1.4964376]

\section{INTRODUCTION}

Wendelstein 7-X (W7-X) - a new fusion experiment of stellarator type - has been successfully commissioned in 2015. Its superconducting coil system provides continuous magnetic field, a prerequisite for proving the steady state capability of this optimized stellarator. The first experimental campaign $^{1}$ started in December 2015 and lasted for three months, featuring $\sim 1000$ plasma discharges. The main purpose of this campaign was the integral commissioning of the machine and diagnostics, which was reached very well. The LCFS of the plasma was defined by 5 poloidal uncooled graphite limiters at the inboard side of the plasma vessel, shaped in a way to conform along its entire length to the edge plasma for the standard iota configuration and to receive the full convective heat load from the plasma. The initially set maximum of $2 \mathrm{MJ}$ (ECR) heating power per discharge has been extended to $4 \mathrm{MJ}$ in the course of OP1.1 because of lower than expected convective heat loads to the limiters. This was caused partially by a significant fraction of the heating power being radiated away from the plasma edge as consequence of strong wall outgassing. The observed plasma parameters exceeded predicted values revealing, e.g., electron temperatures of up to $10 \mathrm{keV}$ (predicted $4 \mathrm{keV}$ ), ion temperatures up to $2 \mathrm{keV}$ and electron densities up to $5 \times 10^{19} \mathrm{~m}^{-3}$. At such high $\mathrm{T}_{\mathrm{e}}$ values of $\sim 10 \mathrm{keV}$ the Thomson scattering (TS) system got close to its detection limit (see Section II B).

More than 20 diagnostic systems have been installed, ${ }^{2}$ commissioned and almost in all cases successfully operated during OP1.1, allowing many physics investigations. This paper gives an overview of the diagnostics with the exception of the neutron counters, ${ }^{3}$ ECRH sniffer probes, ${ }^{4}$ a very comprehensive set of magnetic probes, ${ }^{5}$ a Doppler and a correlation reflectometer, ${ }^{6-8}$ a single line of sight (los) $Z_{\text {eff }}$ measurement and an overview UV-VIS spectrometer which are left out due to space constraints. Some details of the design (the capability of long pulse plasma operation ${ }^{9,10}$ is emphasized if available), the performance of the diagnostics and example results from OP1.1 are presented. In addition the Bayesian inference based Minerva framework used for data analysis of several W7-X diagnostics is briefly discussed.

\section{DIAGNOSTIC SET FOR THE FIRST OPERATION PHASE OF W7-X}

\section{A. Measurement of magnetic flux surfaces}

One of the optimization criteria at W7-X is related to the existence of good closed and nested magnetic flux surfaces in a wide configuration space. In stellarator like magnetic configurations the verification of the existence and quality of magnetic flux surfaces is possible for the vacuum case, i.e., without the existence of a plasma. The diagnostic comprises two $5 \mathrm{~m}$ long vacuum plug-ins with fluorescent swiveling rods in the plasma vessel serving a probing electron beam whose trajectory is optically detected inside the confinement area either due to interaction with background gas in the plasma vessel or due to fluorescence light emission by the electrons hitting the rod. The manipulators can be retracted in the port behind water cooled shutters to sustain steady state plasma discharges.

First flux surface measurements have been performed for the standard OP1.1 magnetic configuration up to a magnetic field of $2.5 \mathrm{~T}$. The experiments confirmed the existence of closed flux surfaces from the magnetic axis up to the limiter. In addition expected intrinsic magnetic island chain at $\mathrm{i}=$ $n / m=5 / 6$ inside the confinement region has been detected. ${ }^{11}$ As modelled by finite element analysis the electromagnetic forces are causing an elastic deformation of the non-planar field coils depending on the field strength, thus influencing the rotational transform and the radial position of that island chain as well. That radial shift of the islands which is of the order of a few $\mathrm{cm}$ has been detected in the experiments. In further experiments (with $i=n / m=1 / 2$ and at low magnetic field of $0.3 \mathrm{~T}$ ) the relative error of the $B_{21}$ Fourier harmonic component was estimated to $<1 \times 10^{-5}$. $^{12}$

\section{B. Diagnostics for plasma profiles}

A multi-pulse Nd:YAG laser Thomson scattering system is installed at W7-X as a powerful diagnostic for electron temperature and density measurements ${ }^{13}$ (for measurement of $T_{e}$ profiles with the XICS diagnostic see Section II D). In OP1.1 a reduced set of 10 fiber bundles was installed to cover a half profile of the plasma cross section, as shown in Fig. 1. The optics is placed in a water cooled immersion tube designed for long pulse operation with a vacuum window protected against coating by a water cooled shutter. Interference filter based polychromators with $\mathrm{Si}$-avalanche diodes and fast digitizers (1 GS/s, 14 bit) are used for detection of the scattered light. The TS system is optimized to measure electron temperatures in the range of $20 \mathrm{eV}-10 \mathrm{keV}$ and electron densities in the range of $2 \times 10^{18}-5 \times 10^{20} \mathrm{~m}^{-3}$ (see Figs. 2 and 3 for comparison of the measured plasma parameters to other diagnostics).

A single channel dispersion interferometer has been installed for measurement of the line integrated density. ${ }^{15}$ It is based on a $20 \mathrm{~W} \mathrm{CO}_{2}$ laser and its second harmonic generated in a $\mathrm{AgGaSe}_{2}$ frequency doubling crystal. Both beams pass the plasma vessel twice being reflected by a corner cube reflector which is located outside the vacuum vessel at an opposite port. A separate reference path is not required. After frequency doubling the residual $10.6 \mu \mathrm{m}$ beam is frequency doubled in a second $\mathrm{AgGaSe}_{2}$ crystal, the phase shift of the $5.3 \mu \mathrm{m}$ signals being detected. Dispersion interferometers are intrinsically insensitive to vibrations along the optical beam path, only vibrations perpendicular to that path increase the 


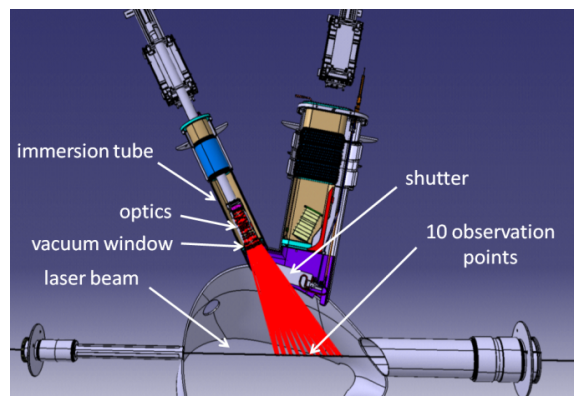

FIG. 1. Main components of the Thomson scattering system.

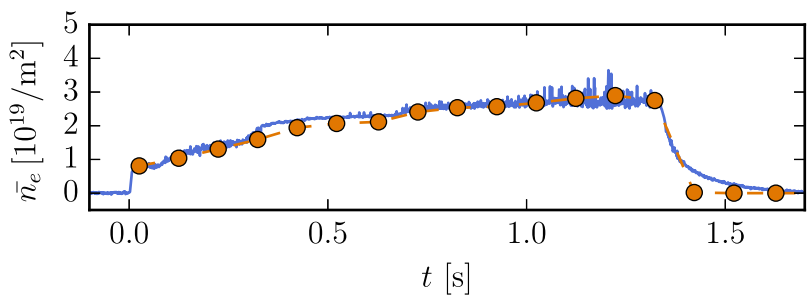

FIG. 2. Time trace of the line integrated electron density measured with the interferometer (blue line) and derived from the Thomson scattering profiles (red dots), pulse 160308007. The last three points of the TS measurement carry no information about the density due to too low electron temperature.

phase noise. The single channel dispersion interferometer shares its sightline with the Nd:YAG laser of the Thomson scattering system, therefore cross-calibration of both systems can be performed easily. Fig. 2 shows a good agreement of the line integrated density measured with the interferometer and derived from the Thomson scattering profiles of the discharge \#160308007.

An Electron Cyclotron Emission diagnostic (ECE) has been operated throughout OP1.1 as the main tool to study electron heating by ECRH and subsequent electron heat transport. It measures the 2 nd harmonic X-mode emission in the frequency band $126 \mathrm{GHz}-162 \mathrm{GHz}$ along a sightline defined by Gaussian optics with a 32-channel heterodyne radiometer located outside the torus hall. ${ }^{16}$ Microwave stray radiation resulting from non absorbed ECRH power is cut out of the spectrum by a $1 \mathrm{GHz}$ wide waveguide Bragg reflection notch filter with depth $>40 \mathrm{~dB}$ showing extremely steep edges and an insertion loss of only $3-5 \mathrm{~dB}$ outside this frequency band. ${ }^{17}$ The radiometer is absolutely calibrated with an overall uncertainty of $\sim 10 \%$. $\mathrm{T}_{\mathrm{e}}$ profiles are derived from the $\mathrm{X} 2$ emission spectra taking into account the finite optical thickness via the ray-tracing code TRAVIS which implies a fully-relativistic model of EC absorption and emission. First comparison with $\mathrm{T}_{\mathrm{e}}$ profiles obtained from Thomson scattering (see example in Fig. 3) and imaging X-ray spectroscopy yields good agreement.

\section{Diagnostics for plasma edge and in-vessel components}

The overview video diagnostic system is mounted in ten tangential ports of W7-X giving a good toroidal coverage. ${ }^{18}$ A dedicated docking mechanism allows fast insertion of each channel in its respective port from outside the machine. Water

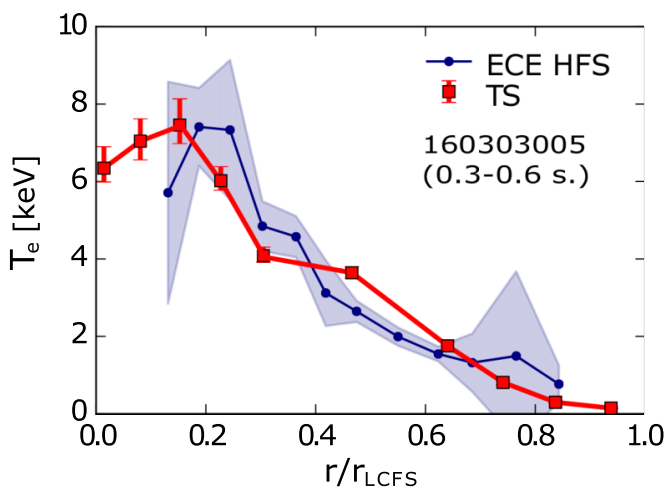

FIG. 3. $\mathrm{T}_{\mathrm{e}}$ profiles from the Thomson scattering and the ECE (high field side emission) diagnostic for pulse \#160303005.

cooled front ends with a pin hole provide sufficient protection from the heat load and coating in long pulse plasma operation. Three different camera types are used. The standard system for regular monitoring of mostly the plasma shape, size and the radiation distribution is equipped with 7 EDICAM cameras ( 400 frames/s @ 1.3 Mpixel). The EDICAM system features FPGA-based real-time image processing and event detection, to be utilized in the forthcoming campaigns of W7-X. 2 PCO PixelFly cameras (12 frames/s @ 1.3 Mpixel) are used for the vacuum magnetic flux surface measurements, and one tangential channel is equipped with an image guide attached to a fast framing camera (Photron SA5, 7 kframes/s @ 1 Mpixel).

The EDICAM system revealed toroidal or poloidal asymmetries as well as the movement of the plasma as reaction to currents applied in trim coils inducing $1 / 1$ magnetic field errors. Filamentary structures at the plasma edge have been detected with the Photron camera, usually at later phases of the discharges accompanied by strong edge radiation or in case of intentional gas injection used for radiative edge cooling experiments. These structures are aligned to the magnetic field lines (Fig. 4), are extended toroidally in the whole observation volume, having in some cases a lifetime of a few milliseconds and revealing poloidal rotation.

The neutral gas density measurement in the range of $2 \times 10^{-7}-1 \times 10^{-2}$ mbar with maximum sampling rate of $2 \mathrm{kHz}$ was performed by five in-vessel pressure gauges of the ASDEX type ${ }^{19}$ and one Penning gauge. ${ }^{20}$ The pressure gauges

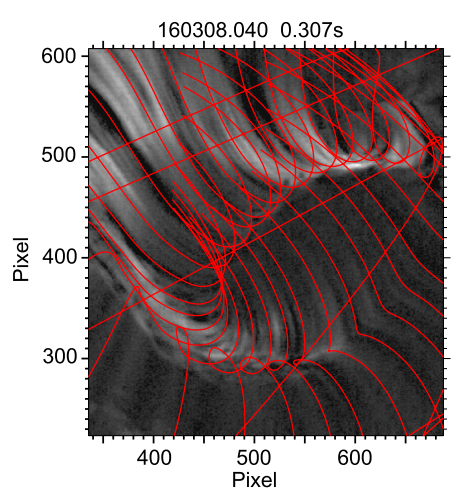

FIG. 4. Short exposure $(\sim 20 \mu \mathrm{s})$ background corrected tangential image showing field line aligned structures. Calculated vacuum field lines are over plotted in red. 


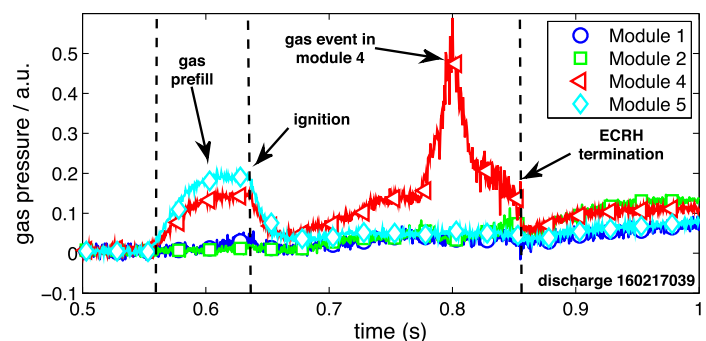

FIG. 5. Neutral gas pressure in four machine modules in a discharge terminated by strong edge radiation.

were placed at front-ends of vacuum plug-ins at the midplane outboard side in all five stellarator modules for investigations of the toroidal symmetry. In Fig. 5 a strong asymmetry of the neutral gas pressure is shown in a discharge terminated by too high edge radiation. Only in module 4 the gas pressure rises from the beginning of the discharge reaching a highly peaked value and is in some cases accompanied by a MARFE-like radiation on the inboard side of module 4 detected by the video cameras.

Several cameras for observation of the limiters and the walls have been installed, among them infrared cameras to measure temperature distribution on the limiter surfaces. Three types of cameras have been used: NIR cameras (measuring at $0.9 \mu \mathrm{m}$, installed in immersion tubes mentioned later in this chapter), one MWIR camera (measuring at 3-5 $\mu \mathrm{m}$, viewing the limiter in module 3 ) and one LWIR camera (measuring at 10-14 $\mu \mathrm{m}$, viewing the limiter in module 5). The data from NIR cameras suffered from plasma emission near the limiter surface. However MWIR and LWIR cameras delivered very useful data. In Fig. 6(a) temperature distribution on the limiter in module 5 measured with the LWIR camera is shown. The topology of magnetic field lines is more complicated than in tokamaks with its 3D structure of inter-woven flux tubes. This is reflected in temperature distribution, which also shows $3 \mathrm{D}$ characteristics.

The MWIR camera shared the same field of view at the limiter in module 3 with a visible camera. ${ }^{21}$ The limiter was seen with high temporal resolution $(\sim 400 \mathrm{~Hz}$ in the IR and $\sim 100 \mathrm{~Hz}$ in the visible) and sub-mm spatial resolution on five of its nine tiles. The filtered visible camera provided absolutely calibrated measurements of carbon and hydrogen light, and therefore inputs to particle balance calculations. ${ }^{22}$ The infrared camera was used to derive space and time resolved power fluxes, as well as in a calorimeter mode to infer total energy deposited on the limiter. From the thermographic
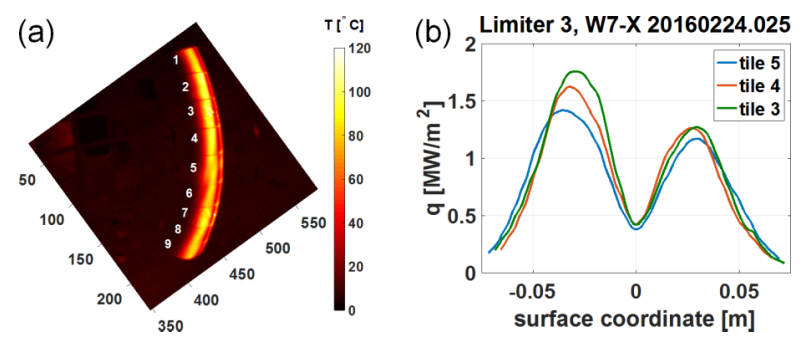

FIG. 6. (a) Infrared image of limiter in module 5 shows heterogeneous temperature distribution due to 3D structure of the scrape-off layer; (b) heat flux density profiles calculated at three tiles of limiter in module 3.

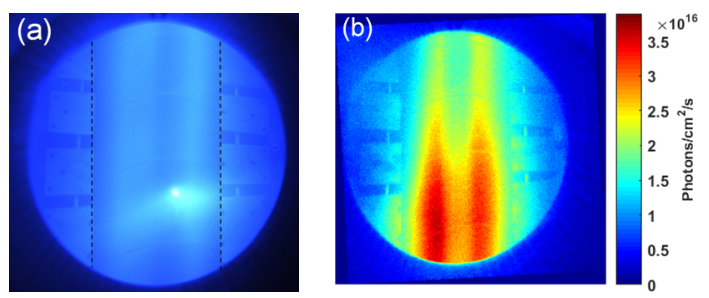

FIG. 7. (a): $465 \mathrm{~nm}$ CIII light showing a carbon bloom observed from a hot spot defect on the limiter in module 3 (black lines indicate the limiter edges). (b): $\mathrm{H}_{\alpha}$ light at the limiter detected by the same camera (discharge \#160308032 at $120 \mathrm{~ms}$ ).

measurements of limiter temperatures, heat flux density has been derived with the help of the THEODOR code. ${ }^{23}$ For a typical discharge of the first campaign with $3 \mathrm{MW}$ of injected power, there is about $1.5 \mathrm{MW} / \mathrm{m}^{2}$ heat flux near the watershed (see Fig. 6(b)). These values are lower than expected due to high fraction of radiated power during the operation which allowed increasing the limit of heating energy from $2 \mathrm{MJ}$ to $4 \mathrm{MJ}$ per plasma pulse.

Fig. 7 shows two limiter images taken with the visible camera in module 3. In Fig. 7(a) a carbon bloom emission from a hot spot defect is shown, and in Fig. 7(b) measured absolute $\mathrm{H}_{\alpha}$ emission at the limiter providing particle fluxes is depicted.

For the observation of the divertor region (in upcoming operation phase) and the limiter in OP1.1 10 immersion tubes with NIR and visible cameras are installed. Each tube is equipped with a set of three cameras, two operating in the visible spectral range with various interference filters $\left(\mathrm{H}_{\alpha}\right.$, CII, and CIII) and one NIR camera. The viewing angle of $86^{\circ}-136^{\circ}$ allows the monitoring of a wide range of the plasma vessel interior with a spatial resolution of $4 \mathrm{~mm}$ (at the optical axis) up to $10 \mathrm{~mm}$ (at the far view) being sufficient for the monitoring purposes. During the first operation phase OP1.1 the visible radiation patterns at the limiters were dominated by hydrogen and carbon emission. In Fig. 8 CIII emission is shown revealing the typical double-stripe structure (compare to $\mathrm{H}_{\alpha}$ emission in Fig. 7(b)).

The IR and visible cameras have been complemented by an absolutely calibrated filterscope system $^{24}$ to obtain spectroscopic data of limiter and first wall recycling and impurity sources with excellent temporal resolution of $10 \mu \mathrm{s}$. A fiber view with a spot size encompassing the width $(16 \mathrm{~cm})$ of the limiter in module 3 provides simultaneous measurements of $\mathrm{HeI}, \mathrm{H}_{\alpha}, \mathrm{H}_{\beta}$, and CII emission. Four

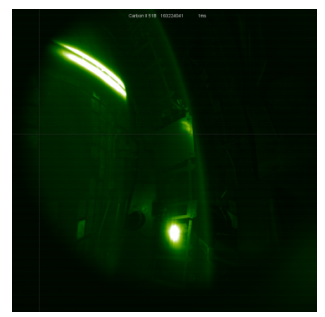

FIG. 8. CIII emission at limiter in module 5 (double-stripe structure at top left) detected with a visible camera and an interference filter at $467 \pm 5 \mathrm{~nm}$. The bright spot at bottom right is due to NeI emission from neon injected by the helium beam injection system for edge cooling experiments. 


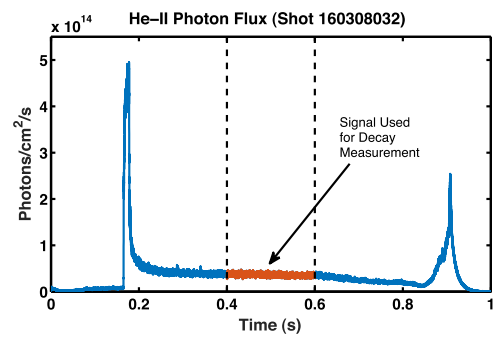

FIG. 9. HeII emission detected by the filterscope viewing the limiter in module 3. $\tau_{\mathrm{p}}{ }^{*} \mathrm{He}$ is derived from the indicated time decay of the emission after a He puff at $\sim 200 \mathrm{~ms}$.

other fiber views of the inboard and outboard wall detected additional $\mathrm{H}, \mathrm{HeI}, \mathrm{HeII}$, and $\mathrm{C}$ emission, as well as visible bremsstrahlung at $523 \mathrm{~nm}$. The HeII emission decay, shown in Fig. 9, was used to determine the $\tau_{\mathrm{p}}{ }^{*}$ (particle confinement time including recycling effects) for helium from a series of perturbative helium puffs that were designed to assess changes in particle confinement. The resulting photon flux from both the visible camera and filterscopes can then be compared to an EMC3-EIRENE synthetic diagnostic ${ }^{25}$ output to infer both the limiter and the wall particle flux and particle balance.

For the gas injection of the thermal helium beam two water cooled vacuum plug-ins capable of steady state plasma operation - one for the upper and one for the lower plasma edge - have been operated during OP1.1. Both were equipped with a valve box with five independent fast piezo valves ${ }^{26}$ positioned only few $\mathrm{cm}$ outside of the LCFS (Fig. 10). The versatile gas injection system has been used for injection of helium for measurement of local edge $n_{e}$ and $T_{e}$ profiles ${ }^{28}$ and effective particle confinement time $\tau_{\mathrm{p}}{ }^{*} \mathrm{He}_{\mathrm{e}}{ }^{22}$ neon for development of a collisional radiative model to extend the parameter range and accuracy of the diagnostic helium beam, ${ }^{28,29}$ argon for impurity transport studies as well as nitrogen for radiative edge cooling. Perpendicular observation of the gas emission at the upper plasma edge was done along 8 los, using quartz fibers and an imaging spectrometer with a CCD camera. ${ }^{28}$

A multipurpose manipulator with a fast reciprocating probe has been assembled at the outboard side near to the equatorial plane. Two probe heads were available: a combined probe measuring the edge plasma profiles or fluctuations (at fixed probe position) as well as a sample station for plasma exposure studies of different materials. The manipulator is able to plunge as far as $350 \mathrm{~mm}$ into the plasma vessel and thereby easily through the LCFS. The combined probe includes two

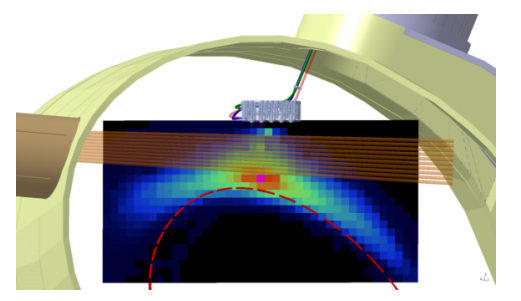

FIG. 10. Geometry of the helium beam diagnostic at the upper plasma edge. Adjustment of the 8 los was supported by the shown EMCR-EIRENE simulation of HeI emission at $706 \mathrm{~nm} .{ }^{27}$ The LCFS is indicated by the dashed red line.

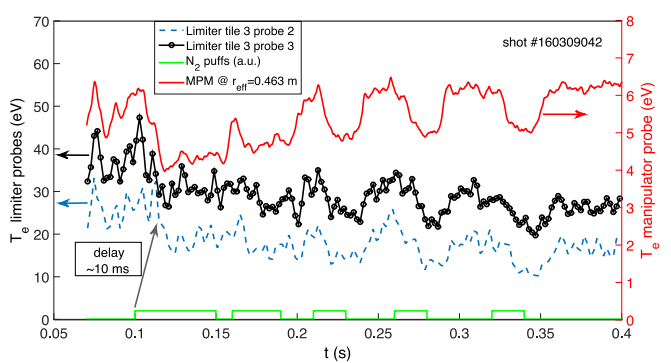

FIG. 11. Cooling of the plasma edge detected with Langmuir probes at different positions in the plasma vessel as reaction on $\mathrm{N}_{2}$ injection into the edge plasma.

magnetic pick-up coil arrays, Langmuir probes in triple probe configuration, and a Mach setup. This allows measuring at the same time and location, the edge radial profiles of the magnetic fields, the electron temperature and density, the electric fields and the plasma flows. ${ }^{30}$ The limiter Langmuir probes comprise two arrays with 20 probes each installed in two tiles of the limiter in module 5 . The probes provided different measures at $2 \mathrm{~ms}$ time resolution at the limiter surface. They measured either the floating potential and the ion saturation current or have swept the voltage for measurement of $n_{e}$ and $T_{e}$, detecting values up to $1 \times 10^{19} \mathrm{~m}^{-3}$ and $5-70 \mathrm{eV}$, respectively. Fig. 11 shows a result of plasma edge cooling by pulsed $\mathrm{N}_{2}$ puffs through the helium beam injection system at the lower plasma edge in module 3 . There is a clear correlation of the injection time and the $\mathrm{T}_{\mathrm{e}}$ values measured both by the manipulator probe (at fixed position in this experiment) in module 4, and by some probes at the limiter (measuring outside of the LCFS) in module 5 . The indicated time delay of $\sim 10 \mathrm{~ms}$ is at least partially due to the opening time of the piezo valve.

\section{Diagnostics for core impurities}

The imaging spectrometer systems X-ray Imaging Crystal Spectrometer $(\mathrm{XICS})^{31-33}$ and High Resolution X-ray Imaging Spectrometer (HR-XIS) ${ }^{34}$ perform complementary measurements of the spectral emission of selected impurities in different charge states, yielding radial profiles of ion and electron temperature, plasma flow velocity, and impurity densities with a time resolution of $5 \mathrm{~ms}$. Both spectrometers have been commissioned during OP1.1.

In those spectrometers, X-rays emitted from the plasma are imaged via a spherical bent crystal onto a $2 \mathrm{D}$ detector. Fig. 12(a) shows typical 2D intensity patterns of ArXVIII and ArXVII emission after injection of trace amounts of argon $\left(n_{A r} / n_{e} \sim 2 \times 10^{-4}\right)$ into the plasma. A spectral fit (green line in Fig. 12(b)) of los integrated, measured spectra (blue line in Fig. 12(b)) and a tomographic inversion yield profiles of above mentioned plasma parameters as shown in Fig. 13. Here, temperature and density profiles have been inferred using an entire forward model of the XICS system within the Minerva Bayesian analysis framework. ${ }^{14}$ First results of the evolution of plasma flow velocity profiles ${ }^{35}$ and argon impurity transport studies ${ }^{36}$ using XICS data are discussed in the given references.

As one of the main impurity diagnostics, the High Efficiency XUV Overview Spectrometer (HEXOS) ${ }^{37-39}$ covers the 

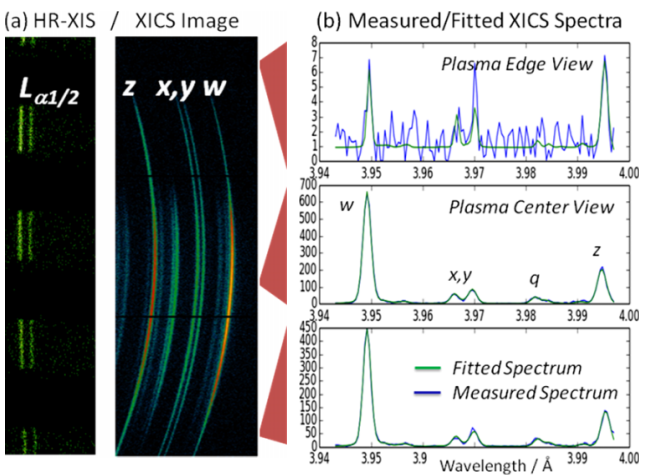

FIG. 12. (a) HR-XIS and XICS raw images of ArXVIII and ArXVII emission. (b) Measured and fitted XICS spectra along los crossing the plasma at the top edge, the center and below the magnetic axis (plots top to bottom, respectively).

wavelength region between 2.5 and $160 \mathrm{~nm}$, distributed over four spectral channels, with high spectral and temporal (up to $1 \mathrm{kHz}$ ). The most intense resonance lines of medium ionization stages of expected intrinsic impurities as well as injected tracer impurities (e.g., Ar, $\mathrm{Ne}, \mathrm{N}$ ) emit light at wavelengths covered by HEXOS.

Fig. 14 shows overview spectra of the lowest energy channel of HEXOS for three different discharge scenarios. Positions of identified emission lines are marked. Clearly identifiable are oxygen and carbon lines in all cases, as well as argon lines for the discharge with argon prefill. All spectra also contain emission lines of fluorine and chlorine, and indications of sulphur.

The Pulse Height Analysis (PHA) system ${ }^{40,41}$ is used for investigation of soft $\mathrm{X}$-ray emission in the energy range from $250 \mathrm{eV}$ to $20 \mathrm{keV}$. It consists of 3 energy channels equipped with Silicon Drift Detectors (SDDs). Each SDD is filtered by a thin foil (Be or Polymer). Each energy channel is equipped with a set of changeable piezo-slits $(0-1.2 \mathrm{~mm})$ and additional exchangeable $\mathrm{Be}$ foils $(10-500 \mu \mathrm{m})$ to assure appropriate flux of X-rays under different discharge conditions. The main purpose of the diagnostic is to identify spectral lines emitted

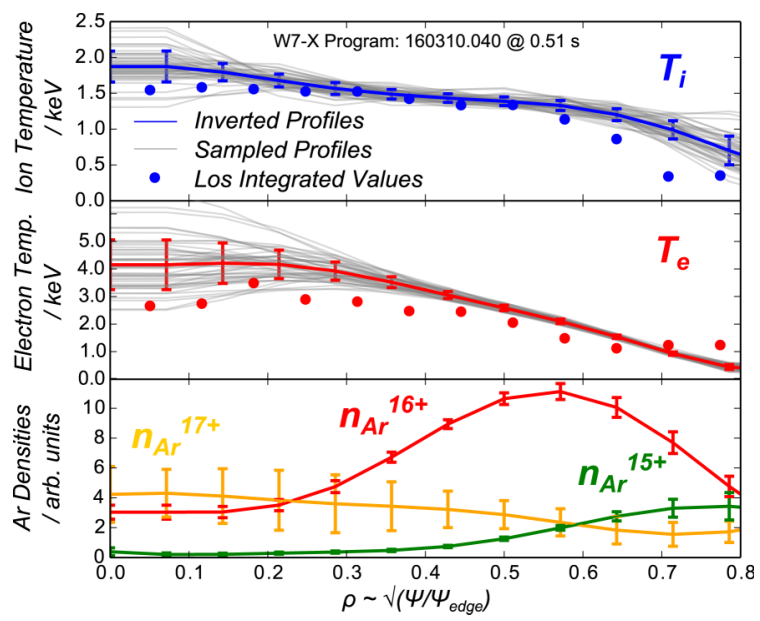

FIG. 13. Inferred local ion and electron temperature (thick lines) including samples from posterior probability distribution (thin lines) as well as argon density profiles of different charge states; los integrated temperatures are shown with dots.

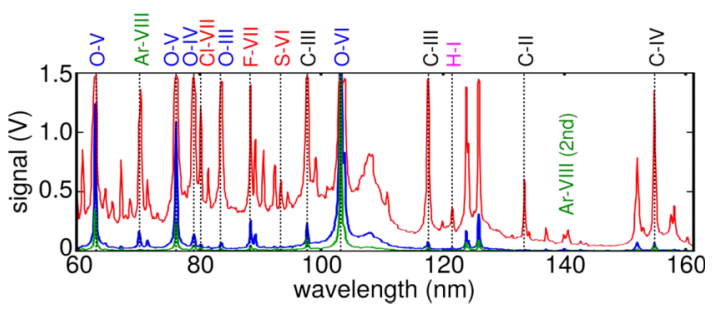

FIG. 14. HEXOS spectra (spectrometer 4) of three different discharge scenarios (green: no argon prefill, blue: with argon prefill, red: with argon prefill during radiation collapse).

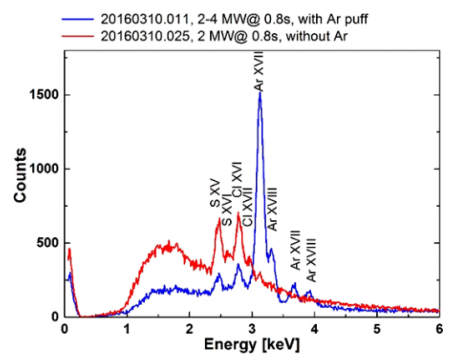

FIG. 15. Integrated spectra from two discharges with (blue) and without (red) argon prefill (same as in Fig. 14).
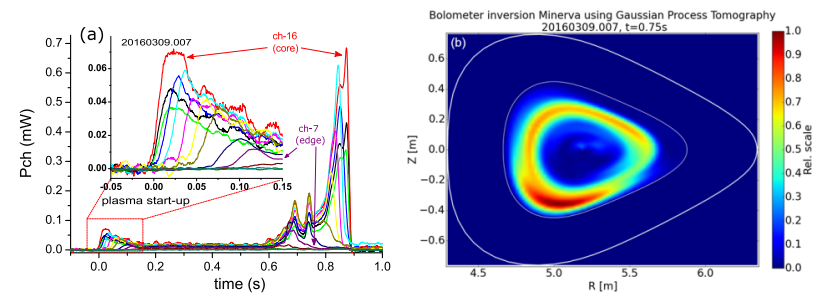

FIG. 16. (a) Time traces of the total radiation power detected by selected channels of the horizontal bolometer camera. (b) Tomographic inversion of the signals shown in (a) using Gaussian Process Tomography. ${ }^{44}$

by impurities in the plasma ${ }^{39}$ as well as estimate the electron temperature and suprathermal electron distribution from the continuum radiation. During OP1.1 the PHA system was launched and tested. Two example spectra detected by the channel with 8 and $25 \mu \mathrm{m}$ Be foil are presented in Fig. 15.

Two endoscopes with horizontal and vertical bolometer cameras based on metal-resistive detectors have been operated during OP1.1. ${ }^{42,43}$ The aperture plates and detector holders are water cooled as protection from thermal overheating as well as to minimize thermal drifts of the detector offset. Metal meshes mounted in front of the detectors in addition to ceramic absorber layers coated on the inner side of the detector enclosures suppress the EC stray radiation in the detector chamber by factor of 300 . Pneumatically driven shutters protect the detectors from contamination during machine conditioning as well as for offset measurements and in situ calibrations even during long pulse plasma operation.

During OP1.1, no microwave interference on the detected signals has been observed. Total radiative power losses from the plasma of $25 \%-40 \%$ of the heating power (being varied in different experiments) have been derived. Fig. 16 shows signals from single bolometer channels and reconstructed 2D emission using the Minerva framework of a hydrogen plasma with a strong radiation zone at the outer radial region. 


\section{E. The Minerva framework}

Minerva is a generic software framework for Bayesian scientific modeling and data analysis. ${ }^{14}$ It uses the concept of Bayesian graphical models ${ }^{45}$ to fully describe forward models, statistical and systematic uncertainties, regularizing priors and their dependencies on each other. Several hundred modules (nodes in the graphical models) have been written (e.g., for the equilibrium, Biot-Savart, bremsstrahlung, los integration). About 20 diagnostic systems have been modeled and used at JET, MAST, TJ-II, TCV, KSTAR, ASDEX Upgrade and W7-X, making Minerva the default diagnostic modeling and analyzing system at W7-X. The Bayesian approach in combination with the Minerva architecture allows multiple diagnostics to be easily combined to give evidence on physics parameters (such as a density profile) from multiple diagnostics jointly, which can significantly increase the accuracy of reconstructions. ${ }^{46,47}$ An important aspect of the framework is the separation of modeling from generic inference algorithms such as linear and nonlinear optimization or Markov Chain Monte Carlo for exploration of the posterior probability distribution. Minerva is able to call legacy codes (for example for 3D equilibria or ECE ray tracing) as separate services running on a virtual cloud using a service oriented architecture. $^{14,48}$

\section{SUMMARY}

The optimized superconducting stellarator Wendelstein $7-X$ has been successfully commissioned in 2015. The first experimental campaign with five uncooled graphite inboard limiters defining the LCFS has been conducted between December 2015 and March 2016 allowing the integral commissioning of all machine components and installed diagnostics. More than 20 diagnostic systems have been put into operation and delivered in almost all cases reliable measurements allowing many physics investigations in $\sim 1000$ discharges of up to $6 \mathrm{~s}$ length and with plasma temperatures and densities exceeding predicted values from simulations.

\section{ACKNOWLEDGMENTS}

This work has been carried out within the framework of the EUROfusion Consortium and has received funding from the Euratom research and training programme 2014-2018 under Grant Agreement No. 633053. The views and opinions expressed herein do not necessarily reflect those of the European Commission. This work was funded in part by the Department of Energy under Grant No. DE-SC0014210. This scientific work was also partly supported by Polish Ministry of Science and Higher Education within the framework of the scientific financial resources in the years 2015 and 2016.

\footnotetext{
${ }^{1}$ T. Sunn Pedersen et al., Nucl. Fusion 55, 126001 (2015).

${ }^{2}$ R. König et al., J. Instrum. 10, P10002 (2015).

${ }^{3}$ B. Wiegel et al., Radiat. Prot. Dosim. 161, 326 (2014).
}

${ }^{4}$ D. Moseev et al., Rev. Sci. Instrum. 87, 083505 (2016).

${ }^{5}$ M. Endler et al., Fusion Eng. Des. 100, 468 (2015).

${ }^{6} \mathrm{M}$. Hirsch et al., in First EPS Conference on Plasma Diagnostics (Proceedings of Science, 2015), p. 111.

${ }^{7}$ T. Windisch et al., in 42nd EPS Conference on Plasma Physics (EPS, 2015), Vol. 39E.

${ }^{8}$ A. Kraemer-Flecken et al., Rev. Sci. Instrum. 81, 113502 (2010).

${ }^{9}$ H.-J. Hartfuss, R. König, and A. Werner, Plasma Phys. Controlled Fusion 48, R83 (2006).

${ }^{10}$ R. König et al., Rev. Sci. Instrum. 83, 10D730 (2012).

${ }^{11}$ M. Otte et al., Plasma Phys. Controlled Fusion 58, 064003 (2016).

${ }^{12}$ T. Sunn Pedersen et al., "Confirmation of the topology of the Wendelstein $7-$ X magnetic field to better than 1:100,000," Nature Comm. (to be published).

${ }^{13}$ E. Pasch et al., Rev. Sci. Instrum. 87, 11E729 (2016).

${ }^{14} \mathrm{~J}$. Svensson and A. Werner, in International Symposium on Intelligent Signal Processing-WISP (IEEE, 2007), Vol. 955.

${ }^{15} \mathrm{P}$. Kornejew, H. Trimino Mora, and M. Hirsch, "Single channel interferometer for the stellarator Wendelstein 7-X," Rev. Sci. Instrum. (unpublished).

${ }^{16} \mathrm{M}$. Hirsch et al., in 1st EPS Conference on Plasma Diagnostics, Frascati, Rome, Italy, 2015.

${ }^{17}$ D. Wagner et al., J. Infrared, Millimeter, Terahertz Waves 32, 1424 (2011).

${ }^{18}$ G. Kocsis et al., Fusion Eng. Des. 96-97, 808 (2015).

${ }^{19} \mathrm{G}$. Haas and H.-S. Bosch, Vacuum 51, 39 (1998).

${ }^{20} \mathrm{~K}$. Flesch et al., Rev. Sci. Instrum. 87, $11 \mathrm{E} 529$ (2016).

${ }^{21}$ G. A. Wurden, L. A. Stephey, C. Biedermann, M. W. Jakubowski, J. P. Dunn, M. Gamradt, and W7-X Team, Rev. Sci. Instrum. 87, 11D607 (2016).

${ }^{22}$ L. A. Stephey et al., Rev. Sci. Instrum. 87, 11D606 (2016).

${ }^{23}$ A. Herrmann, W. Junker, K. Günther, S. Bosch, M. Kaufmann, J. Neuhauser, G. Pautasso, T. Richter, and R. Schneider, Plasma Phys. Controlled Fusion 37, 17 (1995).

${ }^{24}$ R. J. Colchin et al., Rev. Sci. Instrum. 74, 3 (2003).

${ }^{25} \mathrm{H}$. Frerichs et al., Rev. Sci. Instrum. 87, 11D441 (2016).

${ }^{26} \mathrm{M}$. Griener et al., "Fast piezoelectric valve for controlled gas injection in magnetically confined fusion plasmas," Rev. Sci. Instrum. (submitted).

${ }^{27} \mathrm{~F}$. Effenberg et al., "Numerical investigation of plasma edge transport and limiter heat fluxes in Wendelstein 7-X startup plasmas with EMC3-Eirene," Nucl. Fusion (submitted).

${ }^{28}$ T. Barbui et al., Rev. Sci. Instrum. 87, 11E554 (2016).

${ }^{29}$ M. Krychowiak, M. Brix, D. Dodt, Y. Feng, R. Koenig, O. Schmitz, J. Svensson, and R. Wolf, Plasma Phys. Controlled Fusion 53, 035019 (2011).

${ }^{30} \mathrm{P}$. Drews et al., in $43 r d$ EPS Conference on Plasma Physics, 2016.

${ }^{31}$ N. A. Pablant et al., in 41 st EPS Conference on Plasma Physics (EPS, 2014), Vol. 38F.

${ }^{32}$ A. Langenberg, J. Svensson, H. Thomsen, O. Marchuk, N. A. Pablant, R. Burhenn, and R. C. Wolf, Fusion Sci. Technol. 69, 560 (2016).

${ }^{33} \mathrm{~A}$. Langenberg et al., in 41 st EPS Conference on Plasma Physics (EPS, 2014), Vol. 38F.

${ }^{34} \mathrm{G}$. Bertschinger et al., Rev. Sci. Instrum. 75, 3727 (2004).

${ }^{35}$ N. A. Pablant et al., in 43rd EPS Conference on Plasma Physics, 2016.

${ }^{36}$ A. Langenberg et al., in 43rd EPS Conference on Plasma Physics, 2016.

${ }^{37}$ W. Biel, G. Bertschinger, R. Burhenn, R. König, and E. Jourdain, Rev. Sci. Instrum. 75, 3268 (2004).

${ }^{38}$ W. Biel, A. Greiche, R. Burhenn, E. Jourdain, and D. Lepere, Rev. Sci. Instrum. 77, 10F305 (2006).

${ }^{39} \mathrm{H}$. Thomsen et al., J. Instrum. 10, P10015 (2015).

${ }^{40}$ M. Kubkowska et al., J. Instrum. 10, P10016 (2015).

${ }^{41}$ S. Jabłonski, J. Instrum. 10, P10021 (2015).

${ }^{42}$ D. Zhang et al., Rev. Sci. Instrum. 81, 10E134 (2010).

${ }^{43}$ D. Zhang et al., in 38th EPS Conference on Plasma Physics (EPS, 2011), Vol. 35G.

${ }^{44}$ J. Svensson, "Nonparametric tomography using Gaussian processes," JET Internal Report No. EFDA-JET-PR(11) 24, 2011.

${ }^{45}$ S. L. Lauritzen, Graphical Models, Oxford Statistical Science Series (Clarendon Press, 1996).

${ }^{46}$ O. Ford et al., in 36th EPS Conference on Plasma Physics (EPS, 2009), Vol. 33E.

${ }^{47}$ J. Svensson et al., in 37th EPS Conference on Plasma Physics (EPS, 2010), Vol. 34A

${ }^{48}$ A. Werner et al., Fusion Eng. Des. 85, 394 (2010). 